\title{
Quantitative spinal cord MRI in radiologically isolated syndrome
}

Paula Alcaide-Leon, MD, Kateryna Cybulsky, BSc, Stephanie Sankar, BSc, Courtney Casserly, MD, FRCPC, General Leung, PhD, Marika Hohol, MD, FRCPC, Daniel Selchen, MD, FRCPC, Xavier Montalban, MD, PhD, Aditya Bharatha, MD, FRCPC, and Jiwon Oh, MD, PhD, FRCPC

Neurol Neuroimmunol Neuroinflamm March 2018;5:e436. doi:10.1212/NXI.0000000000000436

\section{Abstract \\ Objectives}

To assess whether quantitative spinal cord MRI (SC-MRI) measures, including atrophy, and diffusion tensor imaging (DTI) and magnetization transfer imaging metrics were different in radiologically isolated syndrome (RIS) vs healthy controls (HCs).

\section{Methods}

Twenty-four participants with RIS and $14 \mathrm{HCs}$ underwent cervical SC-MRI on a 3T magnet. Manually segmented regions of interest circumscribing the spinal cord crosssectional area (SC-CSA) between C3 and C4 were used to extract SC-CSA, fractional anisotropy, mean, perpendicular, and parallel diffusivity $\left(\mathrm{MD}, \lambda_{\perp}\right.$, and $\left.\lambda_{\|}\right)$and magnetization transfer ratio (MTR). Spinal cord (SC) lesions, SC gray matter (GM), and SC white matter (WM) areas were also manually segmented. Multivariable linear regression was performed to evaluate differences in SC-MRI measures in RIS vs HCs, while controlling for age and sex.

\section{Results}

In this cross-sectional study of participants with RIS, $71 \%$ had lesions in the cervical SC. Of quantitative SC-MRI metrics, spinal cord MTR showed a trend toward being lower in RIS vs HCs $(p=0.06)$, and there was already evidence of brain atrophy $(p=0.05)$. There were no significant differences in SC-DTI metrics, GM, WM, or CSA between RIS and HCs.

\section{Conclusion}

The SC demonstrates minimal microstructural changes suggestive of demyelination and inflammation in RIS. These findings are in contrast to established MS and raise the possibility that the SC may play an important role in triggering clinical symptomatology in MS. Prospective follow-up of this cohort will provide additional insights into the role the SC plays in the complex sequence of events related to MS disease initiation and progression.

\author{
Correspondence \\ Dr. Oh: \\ ohjiw@smh.ca
}




\section{Glossary}

DMT = disease-modifying treatment; DTI = diffusion tensor imaging; $\mathbf{F A}=$ fractional anisotropy; $\mathbf{G M}=$ gray matter; $\mathbf{H C}=$ healthy control; $\mathbf{M D}=$ mean diffusivity; $\mathbf{M T}=$ magnetization transfer; $\mathbf{M T I}=$ magnetization transfer imaging; $\mathbf{M T R}=$ magnetization transfer ratio; NEX = number of excitation; PSIR = phase-sensitive inversion recovery; RIS = radiologically isolated syndrome; $\mathbf{R O I}$ = regions of interest; $\mathbf{R R M S ~ = ~ r e l a p s i n g - r e m i t t i n g ~ M S ; ~ S C ~ = ~ s p i n a l ~ c o r d ; ~ S C - M R I ~ = ~ s p i n a l ~ c o r d ~ M R I ; ~}$ SC-CSA = spinal cord cross-sectional area; SC-GM = spinal cord gray matter; TE $=$ echo time; $\mathbf{T I}=$ inversion time; $\mathbf{T R}=$ repetition time; $\mathbf{W M}=$ white matter.

Radiologically isolated syndrome (RIS) is a term used to describe neurologically asymptomatic individuals with incidental MRI abnormalities highly suggestive of MS. ${ }^{1}$ It is known that these individuals are at increased risk of eventually developing MS. Recently, a multicenter retrospective study-representing the largest reported study to date in RIS-has found the 5-year risk of conversion to MS to be $34 \% .^{2}$ Despite the recognition that a significant portion of RIS will develop MS, clinical management remains challenging, as there have been no prospective studies assessing the risk of RIS conversion to MS; thus, the natural history of RIS remains unclear. Furthermore, there are no accepted evidence-based management guidelines for RIS, and treatment with a disease-modifying treatment (DMT) indicated for MS is controversial because the benefit of DMTs in RIS has not yet been demonstrated.,

Retrospective studies have identified a number of factors that seem to increase the likelihood of RIS conversion to MS, which include male sex, younger age, and the presence of a spinal cord (SC) lesion on MRI. ${ }^{2,5}$ Among these factors, the presence of an SC lesion portends the greatest risk of conversion to $\mathrm{MS} .^{2}$ In clinical settings, SC lesions are typically assessed in MS using conventional, relaxation-based MRI sequences, which are neither exceedingly sensitive nor specific to pathologic changes in underlying tissue. Using conventional MRI sequences, approximately $35 \%$ of RIS have identifiable asymptomatic SC lesions. ${ }^{2}$

Advanced, quantitative MRI, including diffusion tensor imaging (DTI), and magnetization transfer imaging (MTI) have increased sensitivity to evaluate tissue microstructural properties. DTI-derived parameters measure the magnitude and direction of water diffusion, allowing for improved discrimination between different types of MS-related microstructural abnormalities, including axonal injury and demyelination. ${ }^{6}$ MTI is another quantitative technique that is derived from magnetization exchange between freely mobile protons and those associated with macromolecules such as myelin or axonal membranes. ${ }^{7}$ The most commonly used metric magnetization transfer ratio (MTR) thus reflects myelin and axonal integrity.

Previous studies have demonstrated that quantitative spinal cord MRI (SC-MRI) metrics, including spinal cord crosssectional area (SC-CSA), DTI, and MTR, have increased sensitivity to underlying tissue microstructural changes relevant to clinical disability beyond conventional, lesionbased metrics, and that these measures can provide insight into clinically relevant pathologic processes in $\mathrm{MS} .^{8-13}$ In RIS, brain atrophy has previously been demonstrated, and the presence of SC lesions has been shown to increase the risk of developing MS, ${ }^{2,14}$ but quantitative SC-MRI metrics have not yet been assessed. Accordingly, we hypothesized that there would be differences in quantitative SC-MRI measures in RIS vs healthy controls (HCs), in addition to the presence of $\mathrm{SC}$ lesions. Applying these techniques to the SC in RIS may provide insights into disease mechanisms in the earliest detectable stage of MS, which may provide the needed guidance in managing RIS in day-to-day clinical practice.

\section{Methods}

\section{Standard protocol approvals, registrations, and patient consents}

This study was approved by the Institutional Review Board of St Michael's Hospital. All participants provided written informed consent.

\section{Study participants}

Twenty-four participants with RIS were prospectively recruited from the St Michael's Hospital MS clinic by convenience sampling from July 2014 to September 2015. The exploratory nature of this study precluded a sample size calculation from being performed. Inclusion criteria for the RIS group were adult patients ( $\geq 18$ years of age) meeting previously published clinical and MRI criteria for RIS (incidentally identified T2-hyperintense lesions on MRI-brain fulfilling 3 of 4 Barkhof criteria without neurologic symptoms). ${ }^{1}$ Fourteen HCs were recruited from hospital staff and volunteers. HCs with MRI lesions suspicious for demyelination on MRI or other incidental brain or SC imaging findings were excluded from the analysis $(\mathrm{n}=2)$.

\section{Clinical assessment}

Patients with RIS underwent clinical examination to obtain Expanded Disability Status Scale scores by a neurostatuscertified examiner within 30 days of MRI. There were no participants who experienced a clinical relapse or neurologic symptom suggestive of MS between recruitment and study visit. 


\section{MRI}

MRIs were performed on a 3T MRI scanner (Siemens Skyra, Erlangen, Germany) with a 20-channel head-neck coil and a 16-channel spine-array coil.

\section{Brain MRI}

Acquired brain sequences included T1-weighted magnetization-prepared rapid acquisition gradient echo: $\mathrm{TR} / \mathrm{TE} /$ Flip angle $=1,900 \mathrm{~ms} / 2.52 \mathrm{~ms} / 9^{\circ}$, parallel acceleration technique mode $=$ GRAPPA, acceleration factor $=2$, slice thickness $=1 \mathrm{~mm}$, field-of-view $($ FOV $)=250 \mathrm{~mm}$, inplane resolution $=1 \times 1 \mathrm{~mm}^{2}$, and number of slices $=176$ and T2-weighted fluid-attenuated inversion recovery: TR/TE $=$ $4,800 \mathrm{~ms} / 353 \mathrm{~ms}$, TI $=1,800 \mathrm{~ms}$, parallel acceleration technique mode $=$ GRAPPA, acceleration factor $=2$, slice thickness $=1 \mathrm{~mm}, \mathrm{FOV}=256 \mathrm{~mm}$, in-plane resolution $=1 \times$ $1 \mathrm{~mm}^{2}$, and number of slices $=176$.

\section{Spinal cord MRI}

Acquired SC sequences included magnetization transfer (MT) weighted images: 3D T2*-weighted, gradient-echo sequence with an MT prepulse $(1.5-\mathrm{kHz}$ off-resonance sincgauss-shaped radiofrequency saturation pulse), flip angle/ $\mathrm{TR} / \mathrm{TE}=9^{\circ} / 47 \mathrm{~ms} / 11.2 \mathrm{~ms}$, which yielded 3-mm axial slices (20 contiguous) spanning C3-C4 with FOV $=224 \mathrm{~mm}$, and a nominal in-plane resolution of $0.6 \times 0.6 \mathrm{~mm}^{2}$. $\mathrm{MT}_{\text {off }}$ images used the same parameters but excluded the MT prepulse.

DTI data: cardiac-gated, axial fat-suppressed, high-resolution diffusion-weighted imaging with readout-segmented echoplanar imaging, parallel imaging, and a 2-dimensional navigator-based reacquisition ${ }^{15}$ (RESOLVE) was obtained across C3-C4 in 12 noncoplanar gradient directions. Flip angle $/ \mathrm{TR} / \mathrm{TE}=180^{\circ} / 220 \mathrm{~ms} / 53 \mathrm{~m}, \mathrm{~b}=500$ seconds $/ \mathrm{mm}^{2}$, trigger delay $=0 \mathrm{~ms}$, acquisition window $=420 \mathrm{~ms}$, parallel acceleration technique mode = GRAPPA, acceleration factor $=2$, slice thickness $=3 \mathrm{~mm}, \mathrm{FOV}=150 \mathrm{~mm}$, in-plane resolution $=1.5 \times 1.5 \mathrm{~mm}^{2}$, and slice number $=20$.

Sagittal 2D T1-phase-sensitive inversion recovery (PSIR) of the cervical spine: $\mathrm{FOV}=220 \mathrm{~mm}$; in-plane resolution $=0.7 \times$ $0.7 \mathrm{~mm}$; slice thickness $=3 \mathrm{~mm}$; TR $/ \mathrm{TE}=2,400 / 9.4 \mathrm{~ms}$; TI $=$ $400 \mathrm{~ms}$; averages $=2$; parallel acceleration technique mode $=$ GRAPPA; and acceleration factor $=2$.

\section{Image processing and analysis}

\section{Brain}

Automatic brain lesion segmentation was performed using the Lesion Segmentation Toolbox ${ }^{16}$ for SPM8 (Wellcome Department of Imaging Neuroscience, London, United Kingdom). The optimal initial threshold for segmentation was determined by comparing a reference manual segmentation with automatic segmentations created with different thresholds. Dice coefficients for different thresholds were calculated, and a threshold of 0.25 was selected. Lesion maps were used to fill the segmented lesions in the T1-image with estimated healthy white matter (WM) tissue. ${ }^{17}$ Gray matter (GM), WM, and CSF volumes were obtained using the Voxel-based morphometry 8 (VBM8) toolbox ${ }^{18}$ for SPM8. Lesion, GM, WM, and CSF maps were reviewed for quality control by an experienced neuroradiologist (P.A.-L.). Brain parenchymal fraction (BPF) was calculated by dividing brain parenchymal volume $(\mathrm{GM}+\mathrm{WM})$ by total intracranial volume $(\mathrm{GM}+\mathrm{WM}+\mathrm{CSF})$.

\section{Spinal cord}

$\mathrm{MT}_{\text {on }}$ was registered to $\mathrm{MT}_{\text {off }}$ using a 6 degree-of-freedom, rigid-body process in FLIRT (Oxford Centre for Functional MRI of the Brain's Linear Imaging Registration Tool, Oxford, $\mathrm{UK}) .\left(\mathrm{MT}_{\text {off }}-\mathrm{MT}_{\text {on }}\right) / \mathrm{MT}_{\text {off }}$ was used to calculate MTR (figure e-1 at http://links.lww.com/NXI/A25).

Each diffusion-weighted image was registered to the initial b0 volume using a 6 degree-of-freedom, rigid-body registration in FLIRT using the Java Image Science Toolkit. ${ }^{19}$ The diffusion tensor and maps of DTI indices (fractional anisotropy [FA], mean diffusivity $[\mathrm{MD}]$, perpendicular diffusivity $\left[\lambda_{\perp}\right]$, and parallel diffusivity $\left.\left[\lambda_{\|}\right]\right)$, which were calculated from eigenvalues of the diffusion tensor, were produced (figure e-2, http://links.lww.com/NXI/A26). ${ }^{20}$ The $b_{0}$ image was registered to the $\mathrm{MT}_{\text {off }}$ image using deformable transformation, and the extracted information applied to all diffusion-weighted images. Trilinear interpolation was used for deformable transformation while resampling, and rigid/affine transformation was performed using windowed sinc interpolation.

Manual segmentations of the SC-CSA were performed on the $\mathrm{MT}_{-}$on images, and segmentations of the SC-GM and WM were performed on the MT-off images. Mean SC-CSA and GM and WM areas were calculated across segmented C3-C4. Regions of interest (ROI) were also manually drawn on the FA maps across the C3-C4 segment and transferred to the $\mathrm{MD}, \lambda_{\perp}$, and $\lambda_{\|}$maps to obtain individual DTI indices (figure 1). All segmentations were performed by trained individuals supervised by the neuroradiologist (P.A.-L.). Visual inspection of all slices used for manual segmentation was performed (range, 11-15 slices), and slices were discarded if significant artifact precluded accurate ROI delineation. FA slices (2.6\%), 3.9\% of MTR slices, $20.2 \%$ of $\mathrm{MT}_{\text {off }}$ slices (GM and $\mathrm{WM}$ ), and $0.8 \%$ of $\mathrm{MT}_{\text {on }}$ slices (SC-CSA) were excluded from the analysis.

The presence of SC lesions was assessed on PSIR and MT sequences (figure e-3, http://links.lww.com/NXI/A27). Manual segmentation of SC lesions was performed on the PSIR sequence between $\mathrm{C} 1$ and $\mathrm{C} 7 \mathrm{using}$ a 3D slicer ${ }^{21}$ (http://www.slicer.org) by an experienced neuroradiologist (P.A.-L.) (figure 2).

\section{Statistical analysis}

Statistical calculations were performed using STATA version 11 (StataCorp, College Station, TX). Multivariate linear regression analysis was used to compare quantitative SC-MRI measures in RIS vs HCs, while adjusting for age and sex. Because of the exploratory nature of this study, adjustment for multiple comparisons was not performed. 
Figure 1 Quantitative spinal cord MRI maps and segmentations
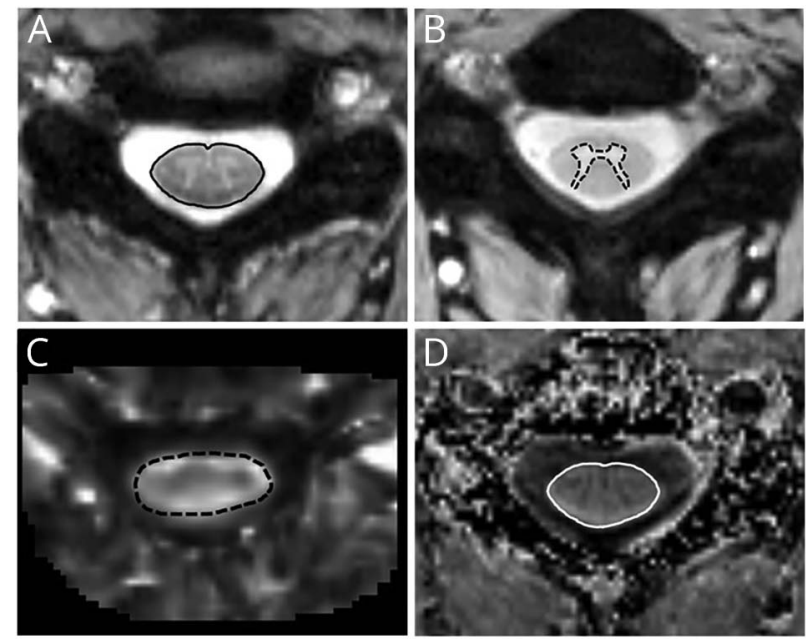

(A) Manual segmentation of the spinal cord cross-sectional area (black line) on high-resolution $\mathrm{T} 2{ }^{*}$-weighted, gradient-echo sequence with magnetizationtransfer (MT) prepulse, (B) manual segmentation of spinal cord gray matter (black dashed line) on T2*-weighted, gradient-echo sequence without MT prepulse, (C) axial cross section of the map of fractional anisotropy with superimposed region of interest (black dashed line), and (D) axial cross section of the map of magnetization transfer ratio with superimposed region of interest (white line).

\section{Results}

This study included a total of 24 participants with RIS and 14 HCs. Forty-eight potential participants with RIS were screened. Reasons for exclusion are shown in figure e-4, http://links.lww. com/NXI/A28. Reasons for which RIS participants had brain MRIs are displayed in table 1 . Participants with RIS were predominantly women (79\%) and had a mean age of 44.6 years, whereas HCs were 60\% women and had a mean age of 36.9 years. Participants with RIS showed a trend toward being older than HCs $(p=0.06)$. None of the RIS participants were treated with DMTs typically used in relapsing-remitting MS (RRMS).

Table 2 summarizes clinical characteristics and MRI measures (volumetrics and quantitative SC-MRI metrics) of the brain and SC in the study population. Seventeen of 24 (71\%) RIS had at least $1 \mathrm{SC}$ lesion, with a mean lesion volume of $0.43 \mathrm{cc}$ (SD, 0.93). The mean brain lesion volume for RIS was $5.28 \mathrm{cc}$ (SD, 6.91).

When quantitative SC-MRI measures were compared between RIS and HCs controlling for age and sex, no significant differences were found with any of the DTI metrics or SC volumetric measures (SC-CSA, spinal cord GM [SC-GM], and spinal cord WM). However, there was a trend toward lower MTR $(p=0.06)$ in RIS vs HCs, and BPF $(p=0.05)$ was lower in RIS vs HCs (figure 3). When quantitative SC-MRI measures were compared in RIS and HCs excluding regions with visible lesions (based on PSIR and MT sequences), there were no detectable differences in any of the quantitative SCMRI measures.
Figure 2 Spinal cord lesions
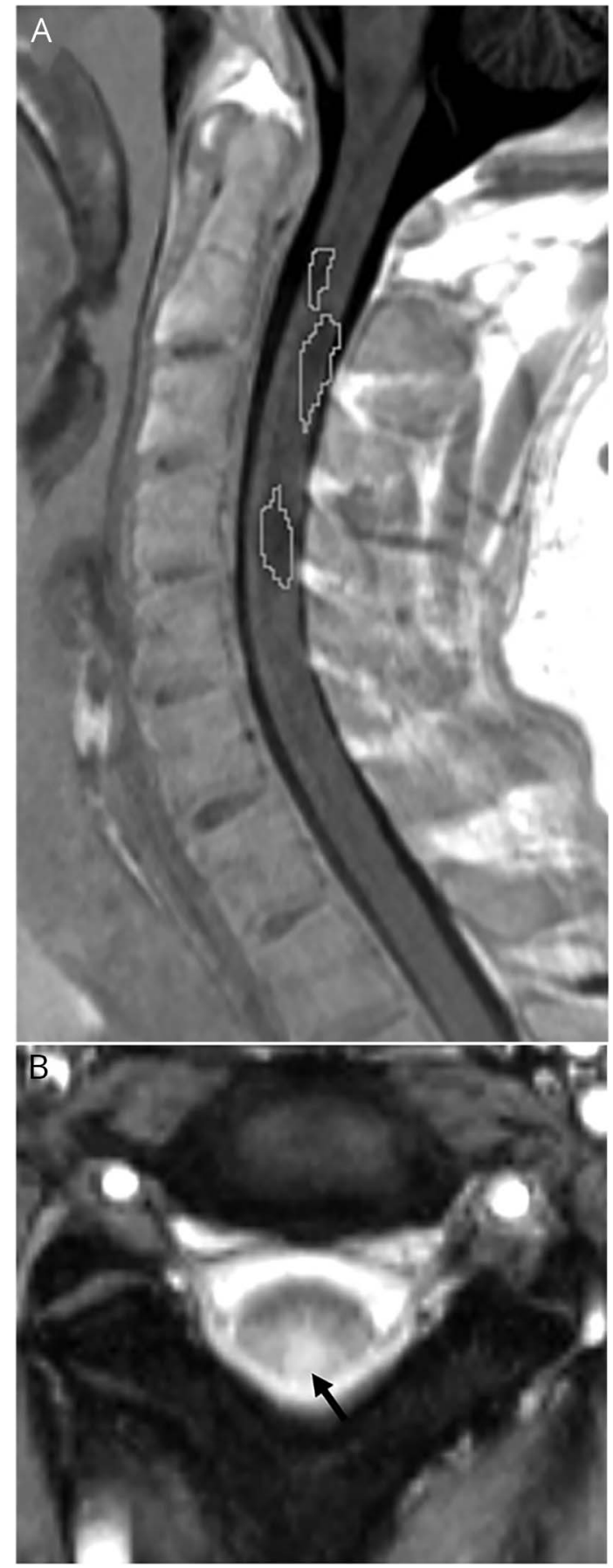

(A) Manual segmentation of cervical spinal cord lesions (white line) in a subject with radiologically isolated syndrome (RIS) on sagittal phase-sensitive inversion recovery sequence and (B) axial magnetization transfer sequence showing a lesion of the same subject with RIS involving the posterior aspect of the cord at the C2-C3 level (black arrow).

\section{Discussion}

In this study, we found minimal microstructural differences using a spectrum of quantitative MRI metrics in the SC of RIS, despite most participants having visible lesions. Furthermore, there was no evidence of SC atrophy. The single detectable 
Table 1 Reasons participants with RIS underwent brain MRI

\begin{tabular}{ll}
\hline Reason for MRI & $\begin{array}{l}\text { Number of } \\
\text { participants }\end{array}$ \\
\hline Headache & 7 \\
\hline Syncope & 2 \\
\hline Nonspecific pain & 2 \\
\hline Incidental opthalmologic finding & 2 \\
\hline $\begin{array}{l}\text { Brief, transient multifocal neurologic } \\
\text { evmptoms atypical for a demyelinating }\end{array}$ & 2 \\
\hline Depression & 1 \\
\hline Subjective cognitive complaints & 1 \\
\hline Fatigue & 1 \\
\hline Elevated prolactin & 1 \\
\hline Pituitary microadenoma workup & 1 \\
\hline Nocturnal tremor & 1 \\
\hline Transient urinary retention & 1 \\
\hline Sinusitis & 1 \\
\hline $\begin{array}{l}\text { Hearing loss later deemed to be medication } \\
\text { induced }\end{array}$ & 1 \\
\hline
\end{tabular}

Abbreviation: RIS = radiologically isolated syndrome.

difference in the SC was a trend toward lower MTR in RIS vs $\mathrm{HCs}$, whereas there was already evidence of brain atrophy in RIS. To our knowledge, this is the first prospective study that has applied a spectrum of quantitative MRI measures to the SC in a prospective cohort of RIS participants and provides valuable insights into early pathologic mechanisms in the SC in MS.

Our findings of a lack of atrophy and minimal microstructural change in the SC in RIS based on quantitative MRI metrics are in contrast to previous studies in clinically definite MS, in which SC quantitative MRI measures, including both DTIand MTI-derived metrics, as well as SC-CSA, GM, and WM were found to be significantly altered in both relapsingremitting and progressive subtypes of MS. This suggests that there is clear microstructural damage in both early and late stages of MS. ${ }^{11,12,22-27}$ Theoretically, a decrease in FA may indicate loss of axonal integrity, whereas an increase in $\lambda_{\perp}$ and decrease in MTR is suggestive of inflammation and/or demyelination. The one quantitative SC-MRI measure that showed a trend toward a difference in RIS vs HCs was that MTR was lower in RIS, suggesting that inflammation and demyelination may be one of the only microstructural changes detectable in the very earliest stage of MS, which is in keeping with known pathologic mechanisms in MS. ${ }^{28}$ In addition, this finding suggests that inflammation and demyelination are the underlying pathologic substrates of visible lesions in the SC in RIS. These findings suggest that there is a temporal sequence of events that lead to MS, with overt microstructural damage of the SC occurring later in the disease course of RIS/MS, after significant pathologic changes (including demyelination, inflammation, and axonal degeneration) have already occurred in the brain. In support of this hypothesis, a number of previous pathologic and imaging studies have demonstrated semi-independence of brain and SC degenerative processes, with limited correlations between brain and SC atrophy. ${ }^{29,30}$ Accordingly, in our study, we found a clear trend toward evidence of brain atrophy in RIS vs HCs, which is in keeping with previous studies that have demonstrated atrophy in brain regions of RIS, including whole-brain, cortical GM, and the thalamus. ${ }^{14,31,32}$ In our study, the difference in brain atrophy observed in RIS vs HCs was likely of borderline statistical significance simply because of the small sample size.

Previous retrospective studies in RIS have shown that among a spectrum of clinical and radiologic factors, the presence of an SC lesion is by far, the factor that increases the risk of developing MS over a follow-up period of approximately 6 years by the greatest magnitude. ${ }^{2,5}$ These findings, together with our observations that suggest a temporal sequence of pathologic changes in early MS (RIS), raise the possibility that a specific pathologic change in the SC may be a necessary factor that "triggers" the onset of clinical symptoms in MS. Alternatively, these observations may indicate that there are factors that influence disease susceptibility, making some individuals with lesions manifest with clinical symptoms (either relapses or disability accumulation), whereas others demonstrate only clinical disability progression without relapses and do not develop any clinically detectable symptoms from individual lesions. These interindividual differences may be related to genetic factors that cause some individuals to manifest with more florid inflammation and subsequent neurodegeneration, triggering clinical symptomatology including relapses or neurologic disability accumulation $^{33}$ or interindividual differences in synaptic plasticity and energy metabolism related to mitochondrial dysfunction that alter the threshold of becoming clinically symptomatic. ${ }^{34}$ Individual factors that influence disease susceptibility are an area of intense clinical interest, particularly in MS, which is a disease with substantial heterogeneity. A better understanding of these factors may have significant treatment implications not just in RIS but also in established MS. These findings are thus worthy of further exploration prospectively and in conjunction with genetic assessments.

We found that the SC-CSA was similar in RIS and HCs, as was the SC-GM and WM area, suggesting the absence of SC atrophy. Numerous previous studies have demonstrated SC atrophy in all stages of MS, particularly GM atrophy in early RRMS. ${ }^{9}$ It is worth noting, however, that using a measure relevant to SC volume (SC-CSA) to identify the presence or absence of atrophy is more problematic than the use of brain volumes to measure brain atrophy because of different 
Table 2 Comparisons of clinical characteristics and MRI measures in participants with RIS vs HCs (adjusted for age and sex)

\begin{tabular}{|c|c|c|c|}
\hline & RIS & HCs & $p$-Value \\
\hline Participants, $\mathbf{n}$ & 24 & 14 & \\
\hline Age, mean (SD) & $44.6(11.1)$ & $36.9(12.8)$ & 0.06 \\
\hline Female \% & 79.2 & 60 & 0.59 \\
\hline EDSS score, median (range) & $0(0-2)$ & & \\
\hline Brain parenchymal fraction, mean (SD) & $0.83(0.02)$ & $0.85(0.02)$ & 0.05 \\
\hline Spinal cord cross-sectional area, $\mathrm{mm}^{2}$, mean (SD) & $78.42(10.1)$ & $77.98(9.47)$ & 0.79 \\
\hline Spinal cord WM cross-sectional area, $\mathrm{mm}^{2}$, mean (SD) & $61.2(9.96)$ & $59.32(8.78)$ & 0.92 \\
\hline Spinal cord GM cross-sectional area, $\mathrm{mm}^{2}$, mean (SD) & $17.71(2.06)$ & $17.81(1.68)$ & 0.95 \\
\hline Brain lesion volume, cc, mean (SD) & $5.28(6.91)$ & 0 & $\mathrm{n} / \mathrm{a}$ \\
\hline No. of patients with at least $1 \mathrm{SC}$ lesion (\%) & $17 / 24(71)$ & 0 & $\mathrm{n} / \mathrm{a}$ \\
\hline SC lesion volume, cc, mean (SD) & $0.43(0.93)$ & 0 & $\mathrm{n} / \mathrm{a}$ \\
\hline FA, mean (SD) & $0.67(0.06)$ & $0.69(0.04)$ & 0.20 \\
\hline MD, $\mu \mathrm{m}^{2} / \mathrm{ms}$, mean (SD) & $0.99(0.13)$ & $1.04(0.09)$ & 0.84 \\
\hline$\lambda_{\|}, \mu \mathrm{m}^{2} / \mathrm{ms}$, mean (SD) & $1.94(0.17)$ & $2.02(0.14)$ & 0.45 \\
\hline$\lambda_{\perp}, \mu \mathrm{m}^{2} / \mathrm{ms}$, mean (SD) & $0.61(0.27)$ & $0.55(0.08)$ & 0.49 \\
\hline MTR, mean (SD) & $0.33(0.03)$ & $0.35(0.02)$ & 0.06 \\
\hline FA NASC, mean (SD) & $0.68(0.05)$ & $0.69(0.04)$ & 0.92 \\
\hline MD NASC, $\mu \mathrm{m}^{2} / \mathrm{ms}$, mean (SD) & $0.98(0.14)$ & $1.04(0.09)$ & 0.58 \\
\hline$\lambda_{||}$NASC, $\mu m^{2} / m s$, mean (SD) & $1.95(0.21)$ & $2.02(0.14)$ & 0.76 \\
\hline$\lambda_{\perp}$ NASC, $\mu m^{2} / \mathrm{ms}$, mean (SD) & $0.60(0.29)$ & $0.55(0.08)$ & 0.75 \\
\hline MTR NASC, mean (SD) & $0.34(0.03)$ & $0.35(0.02)$ & 0.23 \\
\hline
\end{tabular}

Abbreviations: $\lambda_{\perp \mid}=$ parallel diffusivity; $\lambda_{\perp}=$ perpendicular diffusivity; EDSS = Expanded Disability Status Scale; FA = fractional anisotropy; GM = gray matter; $\mathrm{HC}=$ healthy control; $\mathrm{MD}=$ mean diffusivity; $\mathrm{MTR}=$ magnetization transfer ratio; NASC = normal-appearing spinal cord; RIS = radiologically isolated syndrome; $\mathrm{SC}=$ spinal cord; $\mathrm{WM}=$ white matter.

anatomic characteristics of these two structures. Because inflammation drives demyelination, which is accepted to be a dominant pathologic mechanism in MS, lesions are edematous for a period, thus falsely increasing the overall volume regionally. In the brain, edematous lesions do not contribute substantially to the overall brain volume because the ratio between lesion volume and normalappearing brain is usually relatively small. By contrast, $\mathrm{SC}$ lesions tend to occupy relatively large portions of the CSA of the SC, ${ }^{35}$ making it possible that an early, active lesion may temporarily distort the SC volume by a significant magnitude. This issue makes using SC atrophy as a marker of neurodegeneration in RIS/MS problematic, given the possible confounding effects of edema and inflammation. In our sample, over $70 \%$ of patients had SC lesions, making it difficult to establish the degree of atrophy of the normal-appearing cord because the combination of atrophy and lesional edema may have resulted in seemingly "normal" values of SC-CSA. However, the fact that we observed minimal microstructural changes in the SC based on quantitative MRI metrics and that there was no evidence of SC-GM atrophy in lesion-free regions of the SC makes the likelihood of true SC atrophy low in our cohort.

The percentage of RIS with cervical SC lesions in our sample $(70 \%)$ is higher than previously reported $(30 \%-42 \%) .{ }^{2,36,37}$ This is likely due to our use of a novel sequence that is T1-weighted for cervical SC lesion detection (PSIR), which has shown to be $46 \%$ more sensitive than conventional sagittal T2-based sequences for the detection of cervical SC lesions. ${ }^{38}$ Adequate detection of SC lesions is essential in RIS, as it is one of the most important independent predictors of symptom onset. ${ }^{2}$ Our results suggest that the incidence of SC lesions in RIS may be markedly higher than previously reported and promote the utility of more advanced T1-based 
Figure 3 Comparisons of quantitative spinal cord MRI measures and brain atrophy in RIS vs. healthy controls (adjusted for age and sex)

A

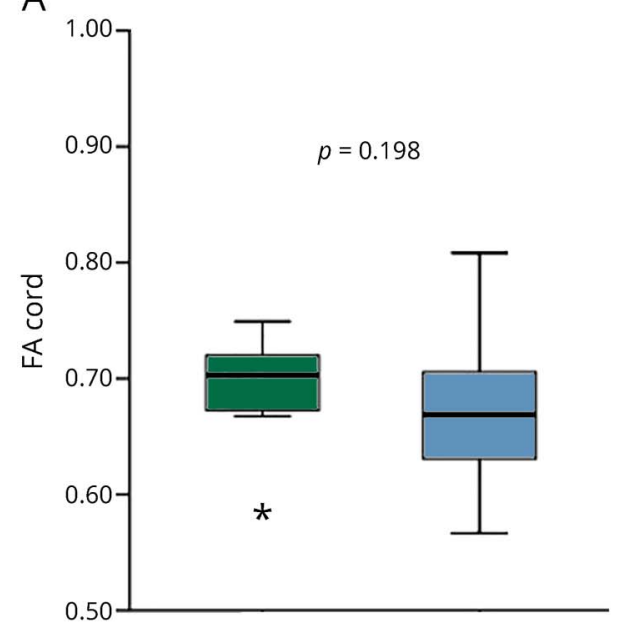

C

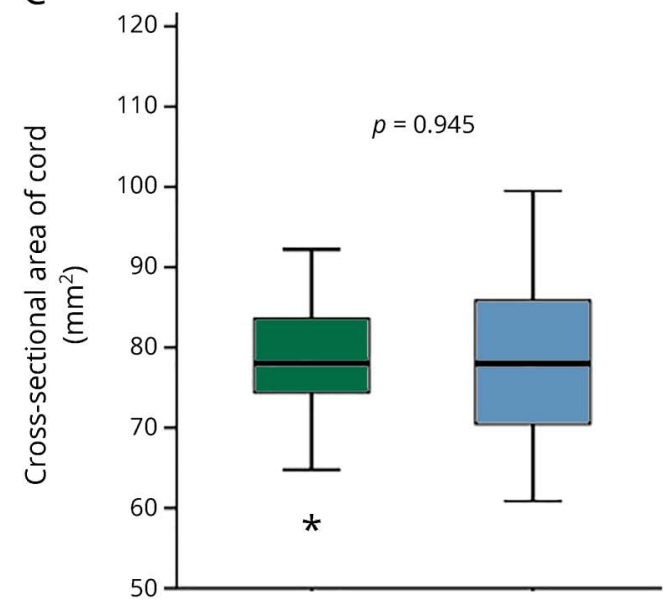

B

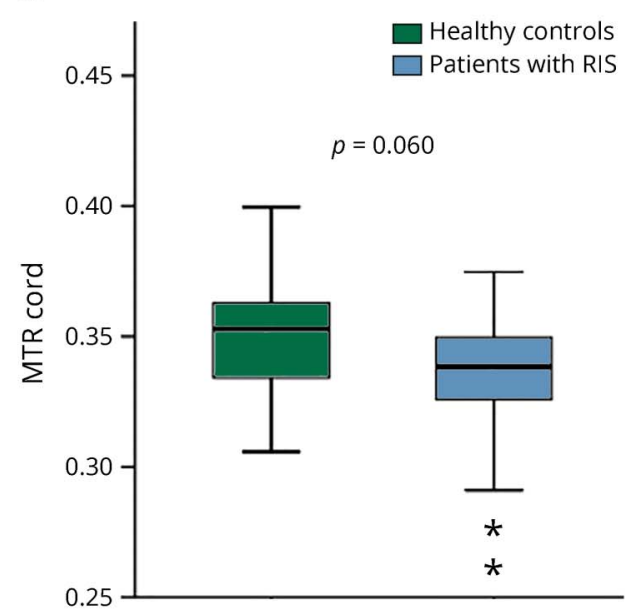

D

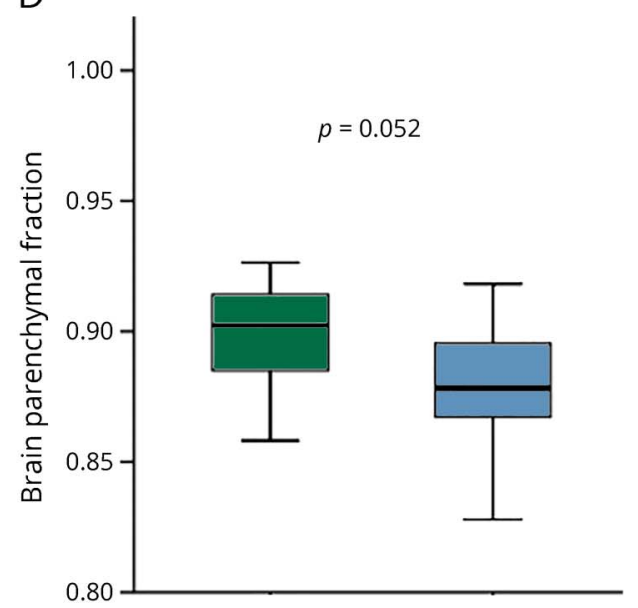

(A) FA cord, (B) MTR code, (C) cross-sectional area of the cord, and (D) brain parenchymal fraction. *Boxplots depict median, interquartile range, and upper and lower fences of each MRI index. Asterisks represent outliers. FA = fractional anisotropy; MTR = magnetization transfer ratio; RIS = radiologically isolated syndrome.

sequences over conventional T2-based sequences to more accurately assess for the presence of SC lesions in both RIS and established MS.

This study has a number of limitations. First, the sample size is relatively small because of the strict inclusion criteria, that RIS is rare, and the prospective study design. Despite the small sample size, we were still able to identify that while most quantitative SC-MRI measures were not different in RIS vs HCs, MTR showed a clear trend toward a difference, which sheds insight into early MS disease mechanisms. Second, we did not correct for multiple comparisons, but given the small sample size and that this was an exploratory study with a priori hypotheses, this is unlikely to be a significant issue. Third, DTI of the SC is technically challenging because of susceptibility, flow, and physiologic motion artifacts. Recent work suggests significant improvements with the use of reduced FOV and increased number of excitations (NEXs). ${ }^{39} \mathrm{Al}-$ though we used a conventional FOV $(150 \mathrm{~mm})$ and NEX, other methods were used to improve the signal-to-noise ratio, including the RESOLVE technique ${ }^{15}$ and cardiac-gating. We also performed careful quality control measures to ensure that data included were of sufficient quality. Finally, despite the apparent utility of quantitative SC measures in investigational settings, because of technical difficulties, measurement variability, and the lack of validation, the clinical applicability of these measures is limited at the current time.

Our findings suggest that in RIS, there are minimal microstructural changes in the SC, with evidence only of demyelination and inflammation. These findings provide needed insight on disease evolution in MS and suggest that 
the SC may play an important role in triggering clinical symptomatology. Furthermore, these findings raise important questions about interindividual susceptibility relating to MS disease progression. Prospective follow-up of this cohort is planned, which will likely provide additional insights into the role the SC plays in the complex sequence of events related to MS disease initiation and progression. Furthermore, practical information that can guide the clinical management of RIS and early MS may be obtained from longitudinal follow-up of this cohort.

\section{Author Contributions}

Conceptualization of the study: Jiwon Oh and Paula AlcaideLeon. Pulse sequence design/implementation and quality control: Paula Alcaide-Leon, Jiwon Oh, Aditya Bharatha, and General Leung. Analysis/interpretation of the data: Paula Alcaide-Leon, Kateryna Cybulsky, Stephanie Sankar, Courtney Casserly, General Leung, Marika Hohol, Daniel Selchen, Xavier Montalban, Aditya Bharatha, and Jiwon Oh. Statistical analysis: Jiwon Oh. Drafting/revising the manuscript: Paula Alcaide-Leon, Kateryna Cybulsky, Stephanie Sankar, Courtney Casserly, General Leung, Marika Hohol, Daniel Selchen, Xavier Montalban, Aditya Bharatha, and Jiwon Oh.

\section{Study Funding}

Multiple Sclerosis Society of Canada Transitional Career Development Award (to J.O.).

\section{Disclosure}

P. Alcaide-Leon received research support from Novartis. K. Cybulsky and S. Sankar report no disclosures. C. Casserly received travel funding from EMD Serono and Novartis. G. Leung holds patents for and receives fees from Wireless Transmission through Faraday Cage, uses of waveguide insert to deliver a wireless signal through the faraday cage, optical detection of multispectral tissue damage, and uses of nearinfrared light source and reflectance spectroscopy to generate images of blood breakdown products using a cell phone; consulted with hospitals for MRI installations and acceptance testing; received research support from the Canadian Institute of Health, National Science \& Engineering Research Council, and S. Michael's Foundation Innovation Fund; and holds stock or stock options from MIMOSA Diagnostics. M. Hohol served on the scientific advisory board of Biogen, EMD Serono, Genzyme, Novartis, Pharmascience Inc, Roche, and Teva Canada Innovation and received travel funding and/or speaker honoraria from Biogen, EMD Serono, Genzyme, and Novartis. D. Selchen received travel funding and/or speaker honoraria from Biogen, Sanofi-GenYme, Teva, Roche, Novartis, and Merk-Serono. X. Montalban served on the scientific advisory board of Actelion, Bayer, Biogen, Merck, Novartis, Oryzon Genomics, Receptos, Roche, SanofiGenzyme, and Teva Pharmaceuticals; received travel funding from Biogen, Merck, Novartis, Receptos, Roche, Sanofi-Genzyme, and Teva Pharmaceuticals; served on the editorial board of Multiple Sclerosis Journal, Journal of Neurology, The International MS Journal, Revista de Neurologia, and
Therapeutic Advances in Neurological Disorders; consulted for Actelion, Bayer, Biogen, Merck, Novartis, Oryzon Genomics, Receptos, Roche, Sanofi-Genzyme, and Teva Pharmaceuticals; and received research support from the Foundation of Barcelona and Fundacio Cemcat. A. Bharatha received travel funding and/or speaker honoraria from EMD Serono, Biogen, and Novartis. J. Oh served on the scientific advisory board of EMD Serono, Genzyme, Novartis, Teva, and Roche; received travel funding and/or speaker honoraria from Roche, Novartis, EMD Serono, and Genzyme; and received research support from Biogen-Idec, the Multiple Sclerosis Society, and the National MS Society. Go to Neurology.org/nn for full disclosure forms.

Received August 13, 2017. Accepted in final form November 6, 2017.

\section{References}

1. Okuda DT, Mowry EM, Beheshtian A, et al. Incidental MRI anomalies suggestive of multiple sclerosis: the radiologically isolated syndrome. Neurology 2009;72:800-805.

2. Okuda DT, Siva A, Kantarci O, et al. Radiologically isolated syndrome: 5-year risk for an initial clinical event. PLoS One 2014;9:e90509.

3. Bourdette D, Simon J. The radiologically isolated syndrome: is it very early multiple sclerosis? Neurology 2009;72:780-781.

4. Bourdette D, Yadav V. The radiologically isolated syndrome revisited: when is it presymptomatic multiple sclerosis? Neurology 2011;76:680-681.

5. Okuda DT, Mowry EM, Cree BAC, et al. Asymptomatic spinal cord lesions predict disease progression in radiologically isolated syndrome. Neurology 2011;76:686-692.

6. Klawiter EC, Schmidt RE, Trinkaus K, et al. Radial diffusivity predicts demyelination in ex vivo multiple sclerosis spinal cords. Neuroimage 2011;55:1454-1460.

7. Schmierer K, Scaravilli F, Altmann DR, et al. Magnetization transfer ratio and myelin in postmortem multiple sclerosis brain. Ann Neurol 2004;56:407-415.

8. Kearney H, Yiannakas MC, Abdel-Aziz K, et al. Improved MRI quantification of spinal cord atrophy in multiple sclerosis. J Magn Reson Imaging 2014;39:617-623.

9. Schlaeger R, Papinutto N, Panara V, et al. Spinal cord gray matter atrophy correlates with multiple sclerosis disability. Ann Neurol 2014;76:568-580.

10. Oh J, Saidha $S$, Chen M, et al. Spinal cord quantitative MRI discriminates between disability levels in multiple sclerosis. Neurology 2013;80:540-547.

11. Agosta F, Absinta M, Sormani MP, et al. In vivo assessment of cervical cord damage in MS patients: a longitudinal diffusion tensor MRI study. Brain 2007;130:2211-2219.

12. Hesseltine SM, Law M, Babb J, et al. Diffusion tensor imaging in multiple sclerosis: assessment of regional differences in the axial plane within normal-appearing cervical spinal cord. AJNR Am J Neuroradiol 2006;27:1189-1193.

13. Filippi $M$, Cercignani $M$, Inglese $M$, et al. Diffusion tensor magnetic resonance im aging in multiple sclerosis. Neurology 2001;56:304-311.

14. Rojas JI, Patrucco L, Miguez J, et al. Brain atrophy in radiologically isolated syn dromes. J Neuroimaging 2015;25:68-71.

15. Porter DA, Heidemann RM. High resolution diffusion-weighted imaging using readout-segmented echo-planar imaging, parallel imaging and a two-dimensional navigator-based reacquisition. Magn Reson Med 2009;62:468-475.

16. Schmidt P, Gaser C, Arsic M, et al. An automated tool for detection of FLAIRhyperintense white-matter lesions in multiple sclerosis. Neuroimage 2012;59: 3774-3783.

17. Pareto D, Sastre-Garriga J, Aymerich FX, et al. Lesion filling effect in regional brain volume estimations: a study in multiple sclerosis patients with low lesion load. Neuroradiology 2016;58:467-474.

18. Ashburner J. A fast diffeomorphic image registration algorithm. Neuroimage 2007;38: 95-113.

19. Rohde GK, Aldroubi A, Dawant BM. The adaptive bases algorithm for intensity-based nonrigid image registration. IEEE Trans Med Imaging 2003;22:1470-1479.

20. Smith SA, Jones CK, Gifford A, et al. Reproducibility of tract-specific magnetization transfer and diffusion tensor imaging in the cervical spinal cord at 3 tesla. NMR Biomed 2010;23:207-217.

21. Fedorov A, Beichel R, Kalpathy-Cramer J, et al. 3D slicer as an image computing platform for the quantitative imaging network. Magn Reson Imaging 2012;30: $1323-1341$

22. Agosta F, Benedetti B, Rocca MA, et al. Quantification of cervical cord pathology in primary progressive MS using diffusion tensor MRI. Neurology 2005;64:631-635.

23. Labiano-Fontcuberta A, Mato-Abad V, Alvarez-Linera J, et al. Normal-appearing brain tissue analysis in radiologically isolated syndrome using 3T MRI. Medicine 2016;95: e4101.

24. Ciccarelli O, Wheeler-Kingshott C, McLean M, et al. Spinal cord spectroscopy and diffusion-based tractography to assess acute disability in multiple sclerosis. Brain 2007; 130:2220-2231.

25. Benedetti B, Rocca MA, Rovaris M, et al. A diffusion tensor MRI study of cervical cord damage in benign and secondary progressive multiple sclerosis patients. J Neurol Neurosurg Psychiatry 2010;81:26-30. 
Filippi M, Bozzali M, Horsfield M, et al. A conventional and magnetization transfer MRI study of the cervical cord in patients with MS. Neurology 2000;54:207.

27. Oh J, Zackowski K, Chen M, et al. Multiparametric MRI correlates of sensorimotor function in the spinal cord in multiple sclerosis. Mult Scler 2013;19:427-435.

28. Chang A, Tourtellotte WW, Rudick R, et al. Premyelinating oligodendrocytes in chronic lesions of multiple sclerosis. N Engl J Med 2002;346:165-173.

29. Cohen AB, Neema M, Arora A, et al. The relationships among MRI-defined spinal cord involvement, brain involvement, and disability in multiple sclerosis. J Neuroimaging 2012;22:122-128.

30. Oh J, Seigo M, Saidha S, et al. Spinal cord normalization in multiple sclerosis. J Neuroimaging 2014;24:577-584.

31. Azevedo CJ, Overton E, Khadka S, et al. Early CNS neurodegeneration in radiologically isolated syndrome. Neurol Neuroimmunol Neuroinflammation 2015;2:e102. doi: 10.1212/NXI.0000000000000102.

32. Giorgio A, Stromillo ML, De Leucio A. Appraisal of brain connectivity in radiologically isolated syndrome by modeling imaging measures. J Neurosci 2015;35:550-558.

33. DeLuca GC, Alterman R, Martin JL, et al. Casting light on multiple sclerosis heterogeneity: the role of HLA-DRB1 on spinal cord pathology. Brain 2013;136:1025-1034.
34. Kantarci $\mathrm{OH}$, Lebrun C, Siva A, et al. Primary progressive multiple sclerosis evolving from radiologically isolated syndrome. Ann Neurol 2016;79:288-294.

35. Tartaglino LM, Friedman DP, Flanders AE, et al. Multiple sclerosis in the spinal cord: MR appearance and correlation with clinical parameters. Radiology 1995;195: 725-732

36. Maia AC Jr, Rocha AJ, Barros BR, et al. Incidental demyelinating inflammatory lesions in asymptomatic patients: a Brazilian cohort with radiologically isolated syndrome and a critical review of current literature. Arq Neuropsiquiatr 2012;70: 5-11.

37. Lebrun C, Le Page E, Kantarci O, et al. Impact of pregnancy on conversion to clinically isolated syndrome in a radiologically isolated syndrome cohort. Mult Scler 2012;18: 1297-1302.

38. Alcaide-Leon P, Pauranik A, Alshafai L, et al. Comparison of sagittal FSE T2, STIR, and T1-weighted phase-sensitive inversion recovery in the detection of spinal cord lesions in MS at 3T. AJNR Am J Neuroradiol 2016;37:970-975.

39. Crombe A, Alberti N, Hiba B, et al. Cervical spinal cord DTI is improved by reduced FOV with specific balance between the number of diffusion gradient directions and averages. AJNR Am J Neuroradiol Epub 2016 Jun 30. 


\title{
Neurology \\ Neuroimmunology \& Neuroinflammation
}

\author{
Quantitative spinal cord MRI in radiologically isolated syndrome \\ Paula Alcaide-Leon, Kateryna Cybulsky, Stephanie Sankar, et al. \\ Neurol Neuroimmunol Neuroinflamm 2018;5; \\ DOI 10.1212/NXI.0000000000000436
}

This information is current as of January 18, 2018

\section{Updated Information \& Services}

References

Citations

Subspecialty Collections

Permissions \& Licensing

Reprints including high resolution figures, can be found at: http://nn.neurology.org/content/5/2/e436.full.html

This article cites 38 articles, 4 of which you can access for free at: http://nn.neurology.org/content/5/2/e436.full.html\#\#ref-list-1

This article has been cited by 4 HighWire-hosted articles: http://nn.neurology.org/content/5/2/e436.full.html\#\#otherarticles

This article, along with others on similar topics, appears in the following collection(s):

All Spinal Cord

http://nn.neurology.org//cgi/collection/all_spinal_cord MRI

http://nn.neurology.org//cgi/collection/mri

Multiple sclerosis

http://nn.neurology.org//cgi/collection/multiple_sclerosis

Information about reproducing this article in parts (figures,tables) or in its entirety can be found online at:

http://nn.neurology.org/misc/about.xhtml\#permissions

Information about ordering reprints can be found online: http://nn.neurology.org/misc/addir.xhtml\#reprintsus

Neurol Neuroimmunol Neuroinflamm is an official journal of the American Academy of Neurology.

Published since April 2014, it is an open-access, online-only, continuous publication journal. Copyright Copyright $\left({ }^{\circ} 2018\right.$ The Author(s). Published by Wolters Kluwer Health, Inc. on behalf of the American Academy of Neurology.. All rights reserved. Online ISSN: 2332-7812.

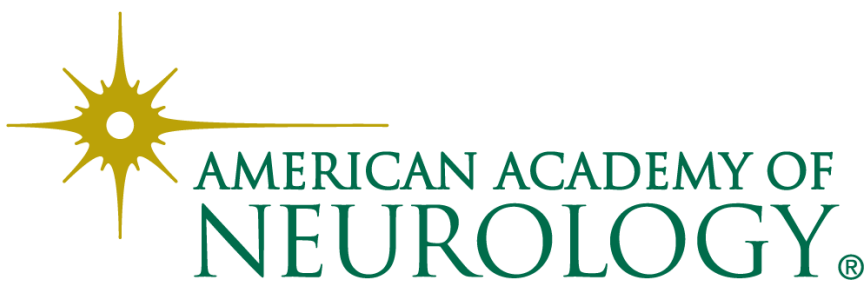

